\title{
Invasive urothelial carcinoma within a calyceal diverticulum associated with renal stones: A case report
}

\author{
TAITO NAKANO $^{1}$, YASUHIDE KITAGAWA ${ }^{1}$, KOUJI IZUMI $^{1}$, HIROKO IKEDA $^{2}$ and MIKIO NAMIKI ${ }^{1}$ \\ ${ }^{1}$ Department of Integrative Cancer Therapy and Urology, Graduate School of Medical Science, \\ Kanazawa University, Kanazawa, Ishikawa 920-8640; ${ }^{2}$ Division of Pathology, Kanazawa \\ University Hospital, Kanazawa University, Kanazawa, Ishikawa 920-8641, Japan
}

Received October 3, 2014; Accepted June 22, 2015

DOI: $10.3892 / \mathrm{ol} .2015 .3590$

\begin{abstract}
Calyceal diverticula are rare outpouchings of the upper collecting system lying within the renal parenchyma. These often contain stones, however, carcinoma within a calyceal diverticulum is uncommon. The present study reports a case of invasive urothelial carcinoma within a calyceal diverticulum associated with renal stones. A 70-year-old male with a left renal mass identified by abdominal computed tomography was referred to the Department of Urology, Kanazawa University Hospital. Pre-operative diagnosis was difficult owing to an atypical imaging finding of a hypovascular renal mass with calcification. A laparoscopic nephroureterectomy was performed, and the surgical specimens showed invasive high-grade urothelial carcinoma within a calyceal diverticulum, and the calcifications were renal stones consisting of $97 \%$ calcium oxalate. Urothelial carcinoma in calyceal diverticula is a rare condition, however, a pre-operative definite diagnosis is difficult and a high potential for invasion of the renal parenchyma is suspected in this disease.
\end{abstract}

\section{Introduction}

Calyceal diverticula are rare outpouchings of the upper collecting system lying within the renal parenchyma, and are found in $0.21-0.6 \%$ of intravenous urograms that are performed on adults (1-4). The majority of patients with calyceal diverticula are asymptomatic, and they are diagnosed when imaging is performed for other reasons (2). Calyceal diverticula often contain stones, and the treatments for urolithiasis should be performed in patients with chronic pain, recurrent urinary tract infection, gross hematuria or decline

Correspondence to: Dr Yasuhide Kitagawa, Department of Integrative Cancer Therapy and Urology, Graduate School of Medical Science, Kanazawa University, 13-1 Takaramachi, Kanazawa, Ishikawa 920-8640, Japan

E-mail: yasukita@med.kanazawa-u.ac.jp

Key words: invasive urothelial carcinoma, calyceal diverticula, renal stone in renal function $(1,2)$. Upper tract urothelial carcinoma is a relatively uncommon disease, accounting for $5 \%$ of all urothelial carcinomas with an annual incidence of 2 cases per 100,000 individuals (5). Due to the low incidence of the disease, mortality rate remains unknown and limited clinical evidence is available regarding treatment (5). Carcinoma within a calyceal diverticulum is highly rare (6-8). The present study reports a case of invasive urothelial carcinoma within a calyceal diverticulum associated with renal stones. It was difficult to make a definitive pre-operative diagnosis owing to atypical imaging findings of the renal mass. Written informed consent was obatined from the patient.

\section{Case report}

In November 2013, a 70-year-old male with diabetes mellitus and hypertension underwent a routine health checkup by a local general practitioner, and a slightly high value of serum carbohydrate antigen (CA)19-9 level (36 U/ml; normal range, $<35 \mathrm{U} / \mathrm{ml}$ ) was found. A left renal mass was detected by abdominal computed tomography (CT) and the patient was accordingly referred to the Department of Intergrative Cancer Therapy and Urology, Graduate School of medical Science, Kanazawa University (Kanazawa, Japan) in January 2014.

Abdominal CT and magnetic resonance imaging revealed a hypovascular renal tumor, $7 \mathrm{~cm}$ in diameter, with calcification (Fig. 1). Retrograde pyelography showed multiple calcifications outside the renal calyx, and a small amount of contrast media accumulated around the calcifications (Fig. 2). Urine cytology from the left pelvis revealed atypical cells. Although no pre-operative definitive diagnosis could be made, these findings could have indicated a renal tumor within a calyceal diverticulum.

A left laparoscopic radical nephroureterectomy by retroperitoneal approach was performed. A massive tumor, $8.0 \times 5.0 \times 4.5 \mathrm{~cm}$ in size, was resected and surgical specimens revealed multiple calcifications that were identified as renal stones consisting of $97 \%$ calcium oxalate and $3 \%$ calcium phosphate (Fig. 3). Microscopically, the tumor was composed of high-grade invasive urothelial carcinoma and squamous differentiated tumor cells extending into the renal cortex (Fig. 4). The definitive pathological diagnosis was of 


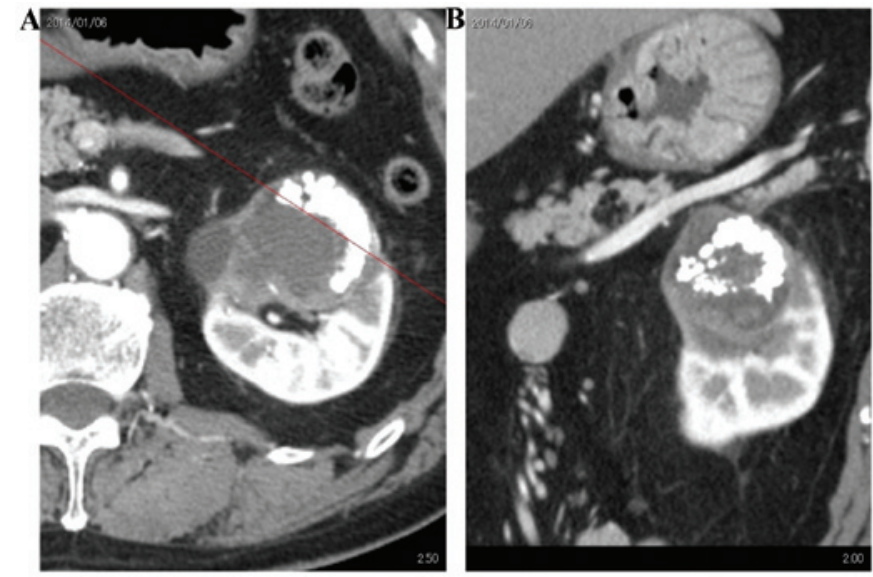

Figure 1. Abdominal computed tomography scan showing a left hypovascular renal mass of $7 \mathrm{~cm}$ in diamater, with calcifications. (A) Axial section. Red line indicates the (B) coronal section.
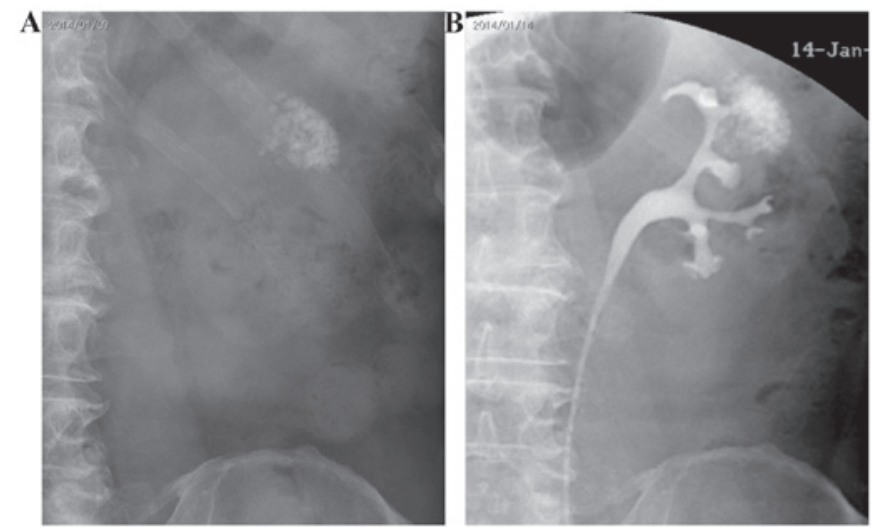

Figure 2. (A) Abdominal X-rays showing multiple calcifications suspected to be left renal stones. (B) Retrograde pyelography showed a small amount of contrast media accumulated around the calcifications outside the renal calyx. No abnormal findings, including filling defects of contrast media, appeared in the renal pelvis.

pT3N0M0 urothelial carcinoma based on the 7th American Joint Committee on Cancer/Union for International Cancer Control tumor-node-metastasis (AJCC/UICC TNM) staging system (9). Administration of tegafur-uracil (300 mg, daily) was begun as adjuvant chemotherapy at two weeks post-surgery and was continued for six months. The serum CA19-9 level normalized after surgery. No recurrent findings were observed during the follow-up period of 12 months.

\section{Discussion}

Urothelial carcinoma within calyceal diverticula is extremely rare, and formation of a definitive diagnosis prior to surgery is difficult (6-8). Zuckerman et al reported a case of a calyceal diverticular urothelial (transitional cell) carcinoma found during percutaneous nephrolithotripsy (6), however, in other cases $(7,8)$, including the present case, the diagnosis was confirmed following radical nephroureterectomy or simple nephrectomy. Cases of urothelial carcinoma associated with renal stones have been reported, however, pre-operative imaging findings of urothelial carcinoma associated with

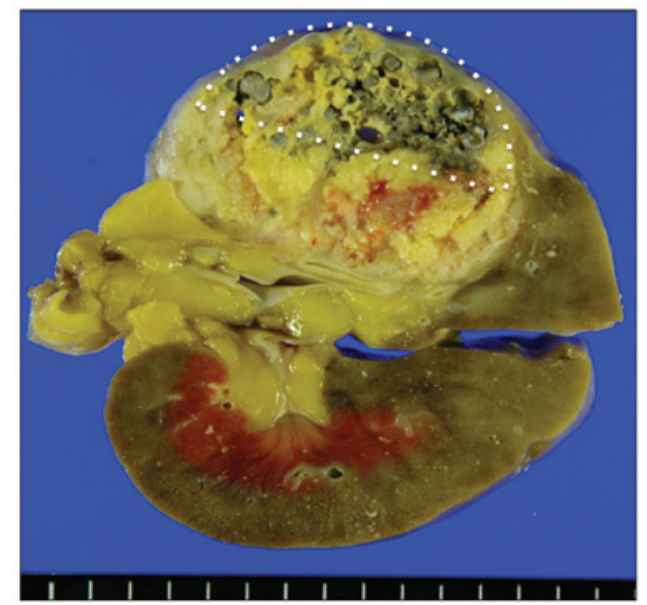

Figure 3. Macroscopic surgical specimen showing a massive renal tumor with multiple renal stones (dotted line).

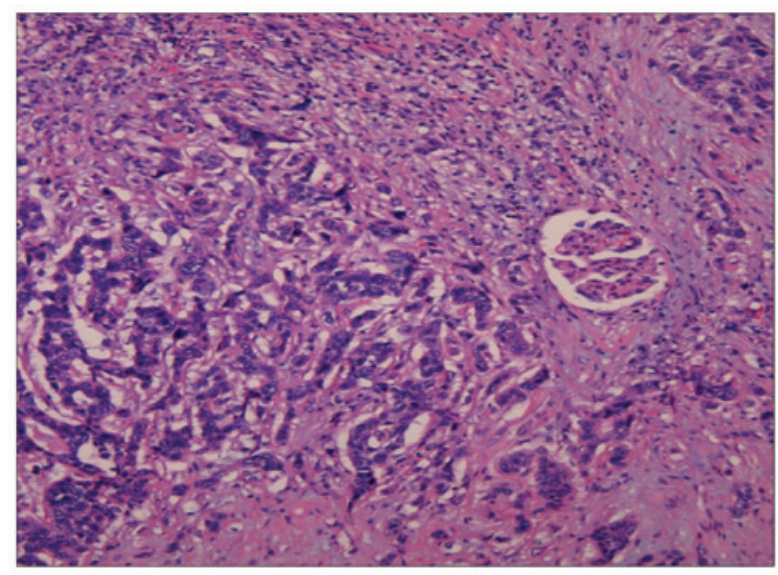

Figure 4. Microscopic appearance of the tumor showing high-grade invasive urothelial carcinoma and squamous differentiated tumor cells extending into the renal cortex (hematoxylin and eosin staining; magnification, x100).

renal stones can complicate the pre-operative diagnosis further (6-8). In one previous study, although malignancy was found in $\sim 50 \%$ of the patients who underwent nephrectomy to treat a stone disease in a non-functioning kidney, the lesion was observed on preoperative imaging in only $29 \%$ of those patients (10). As the mechanism resulting in stone-related urothelial malignancies, chronic irritation and infection may play a significant role in the development of renal pelvis/ureter or bladder cancer (11). There have been several studies on the correlation between squamous cell carcinoma and stones in the urinary tract, and the pathological findings in the present case showed high-grade invasive urothelial carcinoma and squamous differentiated tumor cells. Although the origins of urothelial malignancies associated with stones remain unclear, chronic irritation and infection due to stones may accelerate the differentiation of urothelial carcinoma. As stones can be found in up to $50 \%$ of calyceal diverticula (2), a possible diagnosis of urothelial malignancy should be considered in patients with calyceal diverticula.

Based on the 7th AJCC/UICC TNM staging system (9), the present case was diagnosed as pT3 invasive renal pelvic cancer. An increasing level of attention has recently been focused on 
using a renal pelvic pT3 subclassification to distinguish between microscopic infiltration of the renal parenchyma (pT3a) and macroscopic infiltration or invasion of the peripelvic adipose tissue (pT3b) (12,13). A previous study demonstrated that the 10 -year recurrence-free (pT3a, 58\% vs. pT3b, 38\%; P<0.001) and cancer-specific (pT3a, 60\% vs. pT3b, 39\%; P=0.002) survival rates were decreased in patients with $\mathrm{pT} 3 \mathrm{~b}$ disease (13). Another subclassification has also been proposed: $\mathrm{pT} 3 \mathrm{a}$, in which urothelial carcinoma of the renal pelvis (UCRP) extends only into the renal medulla, and pT3b, in which UCRP extension into the renal cortex is present and/or in which UCRP exhibits peripelvic fat invasion (14). In the study using this second subclassification, the five-year cancer-specific survival rates were 84.6 and $37.3 \%$ for pT3a and pT3b, respectively (14). The present case was diagnosed as pT3b using each of the subclassification systems, and careful follow-up has been indicated.

Deep invasion in urothelial carcinoma within a calyceal diverticulum is an notable clinical problem. In the study by Zuckerman et al, the definite pathological diagnosis in the subsequent laparoscopic radical nephroureterectomy was high-grade transitional cell carcinoma invading the parenchyma (6). Although there is no consensus on the cause of calyceal diverticula, one possible cause is derived from dysfunction within the sphincters surrounding the calyces that facilitate synchronized filling and emptying (2). Such calyceal achalasia results in chronic inefficient emptying, progressive dilatation proximal to the sphincter and subsequent formation of a diverticulum (2). In this situation, possible thinness or loss of the sphincter surrounding the calyceal diverticula mucosa may result in tumor invasion across the sphincter muscle layer. Close examination is indicated in urothelial malignancies within calyceal diverticula in the clinical setting.

In conclusion, this study presented the case of a 70 year old male with invasive urothelial carcinoma within a calyceal diverticulum, that was associated with renal stones. Urothelial carcinoma in calyceal diverticula is a rare condition, and the pre-operative definite diagnosis in the present case was difficult. The current study revealed that this disease exhibits a high potential for invasion of the renal parenchyma. Thus, we hypothesize that invasive urothelial carcinoma should be considered as one of the differential diagnoses in patients with renal masses that are associated with renal stones, which exhibit atypical imaging findings.

\section{References}

1. Timmons JW Jr, Malek RS, Hattery RR and Deweerd JH: Calyceal diverticulum. J Urol 114: 6-9, 1975.

2. Wainganker N, Hayek S, Smith AD and Okeke Z: Calyceal diverticula: A comprehensive review. Rev Urol 16: 29-43, 2014

3. Wulfsohn MA: Pyelocaliceal diverticula. J Urol 123: 1-8, 1980.

4. Michel W, Funke PJ, Tunn UW and Senge T: Pyelocalyceal diverticula. Int Urol Nephrol 17: 225-230, 1985.

5. Oya M and Kikuchi E; Committee for Establishment of Clinical Practice Guideline for Management of Upper Tract Urothelial Carcinoma; Japanese Urological Association: Evidenced-based clinical practice guideline for upper tract urothelial carcinoma (summary - Japanese Urological Association, 2014 edition). Int J Urol 22: 3-13, 2015.

6. Zuckerman JM, Passman C and Assimos DG: Transitional cell carcinoma within a calyceal diverticulum associated with stone disease. Rev Urol 12: 52-55, 2010.

7. Adachi T, Ezaki K and Funai K: A case of transitional cell carcinoma in a pyelocaliceal diverticulum in Japanese. Hinyoukika Kiyo 35: 1383-1386, 1989.

8. Yoshimura K, Yoshida H, Kawase N and Taki Y: A case of transitional cell carcinoma and milk of calcium in a pyelocalyceal diverticulum in Japanese. Hinyoukika Kiyo 44: 649-652, 1998.

9. Sobin L, Gospondarowicz M and Wittekind C (eds): Urological tumours, renal pelvis and ureter. In: TNM Classification of Malignant Tumours. 7th revised edition. Wiley-Blackwell, Hoboken, NJ, pp258-261, 2009.

10. Yeh CC, Lin TH, Wu HC, Chang $\mathrm{CH}$, Chen $\mathrm{CC}$ and Chen WC: A high association of upper urinary tract transitional carcinoma with nonfunctioning kidney caused by stone disease in Taiwan. Urol Int 79: 19-23, 2007.

11. Chow WH, Lindblad P, Gridley G, Nyrén O, McLaughlin JK, Linet MS, Pennello GA, Adami HO and Fraumeni JF Jr: Risk of urinary tract cancers following kidney or ureter stones. J Natl Cancer Inst 89: 1453-1457, 1997.

12. Rouprêt M, Babjuk M, Compérat E, Zigeuner R, Sylvester R, Burger M, Cowan N, Böhle A, Van Rhijn BW, Kaasinen E, et al: European guidelines on upper tract urothelial carcinomas; 2013 update. Eur Urol 63: 1059-1071, 2013.

13. Shariat SF, Zigeuner R, Rink M, Margulis V, Hansen J, Kikuchi E, Kassouf W, Raman JD, Remzi M, Koppie TM, et al: Subclassification of pT3 urothelial carcinoma of the renal pelvicalyceal system is associated with recurrence-free and cancer-specific survival: Proposal for a revision of the current TNM classification. Eur Urol 62: 224-231, 2012.

14. Sassa N, Tsuzuki T, Fukatsu A, Majima T, Kimura T, Nishikimi T, Yoshino Y, Hattori R and Gotoh M: Is pT3 urothelial carcinoma of the renal pelvis a homogeneous disease entity? Proposal for a new subcategory of the pT3 classification. Histopathology 61: 620-628, 2012. 